\title{
Critics of Existent Theory of Mathematical Pendulum Part 1
}

\author{
Zdzisław Pluta, Tadeusz Hryniewicz* \\ Koszalin University of Technology, Racławicka 15-17, 75-620 Koszalin, Poland \\ *E-mail address: Tadeusz.Hryniewicz@tu.koszalin.pl \\ "A proper word to the thing should be given" \\ Cyprian Kamil Norwid
}

\begin{abstract}
In the framework of this paper a deep critics of existent theory of the simple mathematical pendulum is presented. This work consists of two parts. In the first part of the paper, a thesis is stated to derive a mystification character of the theory. The up-to-date, excerpted from the literature, descriptions of the oscillation motion of the mathematical pendulum, are delivered. This part of the paper is to show that the existent theory describes simply a shadow of the rotating apparent mathematical pendulum. The necessity to re-build the existent theory has been indicated.
\end{abstract}

Keywords: Mathematical pendulum; Vibration period; Amplitude; Angular velocity; Force of gravity; Force of inertia

\section{INTRODUCTION}

Evocation of the whole existent knowledge on the mathematical pendulum, even in the form of a condensed matter, has no right justification. The problem is generally known and disseminated so that it may not be repeated in the framework of this elaboration. However, it seems to be worth getting more in-depth to analyze the initial points referred to the pendulum theory of up-to-date knowledge.

It is known that Galileo Galilei (1564-1642), Italian physicist, astronomer and philosopher, was the creator of the pendulum theory [1]. Fundamentals of this theory, being the source, has not changed until today and formulate the same essence.

For many years and centuries, further more or less developed, cognitive structures have been built. All these activities were performed formally, considerably deviating from the cognitive matter. It has been lost the complementary issue of physics and mathematics regarding the phenomenon of harmonic motion. All that did not foster/provide for building an adequate picture of that section of material reality.

A Newtonian approach, reflected for instance in the literature [2-4], has not an adequate character and significantly departs from the reality. More ramified, formalized Lagrange's approach [5] also does not fulfill so essential, basic condition for scientific description of the 
phenomenon of pendulum motion. That description also is not adequate even if its saturation with mathematics is overwhelming.

Existent scientific theory of simple mathematical pendulum yet does not correspond with reality. A conflict is noticed between the theory and reality of the oscillatory pendulum motion. The theory describes the motion of shadow of the oscillatory pendulum which rotates around the vertical axis with a determined angular velocity.

There are only two common elements which connect this inadequate theory with the reality. One of them is time of the body motion in one cycle of this phenomenon. For the rotary motion of this apparent mathematical pendulum it is the time of one complete/full revolution of weight around the vertical axis which is the shadow vibration period of such a pendulum. With the same, it corresponds with the vibration period of a real simple mathematical pendulum that is double time of its motion between the neighbouring turning points.

The second common element is the amplitude. Apparent amplitude of the apparent mathematical pendulum corresponds with this real amplitude, which is the amplitude of vibrations of a real simple mathematical pendulum.

These mentioned in the introduction artifact points of the existent theory will be developed in the paper. One should admit that there is a need and/or necessity to return to the source itself which is the description of the true essence of this oscillation motion. A new theory of the simple mathematical pendulum should be developed. However, it requires a separate elaboration because it is impossible to place so broad merit in one paper. Therefore in this paper only the broad deepen critics of the existent theory of mathematical pendulum has been presented.

\section{MYSTIFICATION THEORY OF FLAT MATHEMATICAL PENDULUM}

Existent theory of the simple mathematical pendulum has a mystification character, being a mental fiction. That is the thesis of the work which will be proven. At the beginning, however, two exemplary descriptions of the oscillation motion of mathematical pendulum will be presented. They differ by the degree of simplification of description, though that artifact's core is analogous. That core has the same nature for all other description versions.

Let us begin from the exemplary description of pendulum motion, excerpted from the literature [2,6]. Reference [6] is devoted for the secondary school students but there also the same knowledge on mathematical pendulum is delivered. Numerous reports on this work (with merit, methodic and merit, didactic and merit, and language) are the main warrants of that non-changeable structure of the grounded theory on the considered pendulum and they have been maintained.

From the formal point of view the definition of mathematical pendulum should be provided. It is stated in reference [2] that the mathematical pendulum (circular) is a body considered as a material point - of mass $m$, hung in a motionless/still point on a nonextensible, weightless (i.e. such which mass may be omitted or put aside) thread of the length $l$ and moving in vertical plane under the action of a force of gravity. That body, deflected/tossed off the position of equilibrium and left for action only the force of gravity makes vibrations/oscillations around of the lowest point - as provided by references on mathematical pendulum.

Reference [5] provides more strict and precise the term of the considered mathematical pendulum, giving the name of simple mathematical pendulum because it performs the 
oscillating motion in one plane, here - vertical plane. Naturally, there is another, spherical mathematical pendulum, also mentioned in that reference. Thus the onomastics has been precised, though it requires some sort of correction. The author of reference [2] says about circular pendulum, quite unnecessarily. There is no justification for that. In turn, speaking about a spherical mathematical pendulum is also non-authorized. The point is rather in a rotary pendulum as such a motion (rotational) is performed by it. Hence, it should be rather a rotary mathematical pendulum.

Now let us illustrate the structure of the flat mathematical pendulum (Fig. 1) with the description of its oscillatory motion. There is the body of mass $m$, hung on a thread/string of the length $l$. The second end of this thread/string is placed/settled in the node/joint. The pendulum is in a sloping position against the perpendicular/vertical one. Its slope/deflection against that position is determined by the angle $\varphi$. The angle of pendulum deflection from vertical to the turning position has been marked by symbol $\varphi^{*}$.

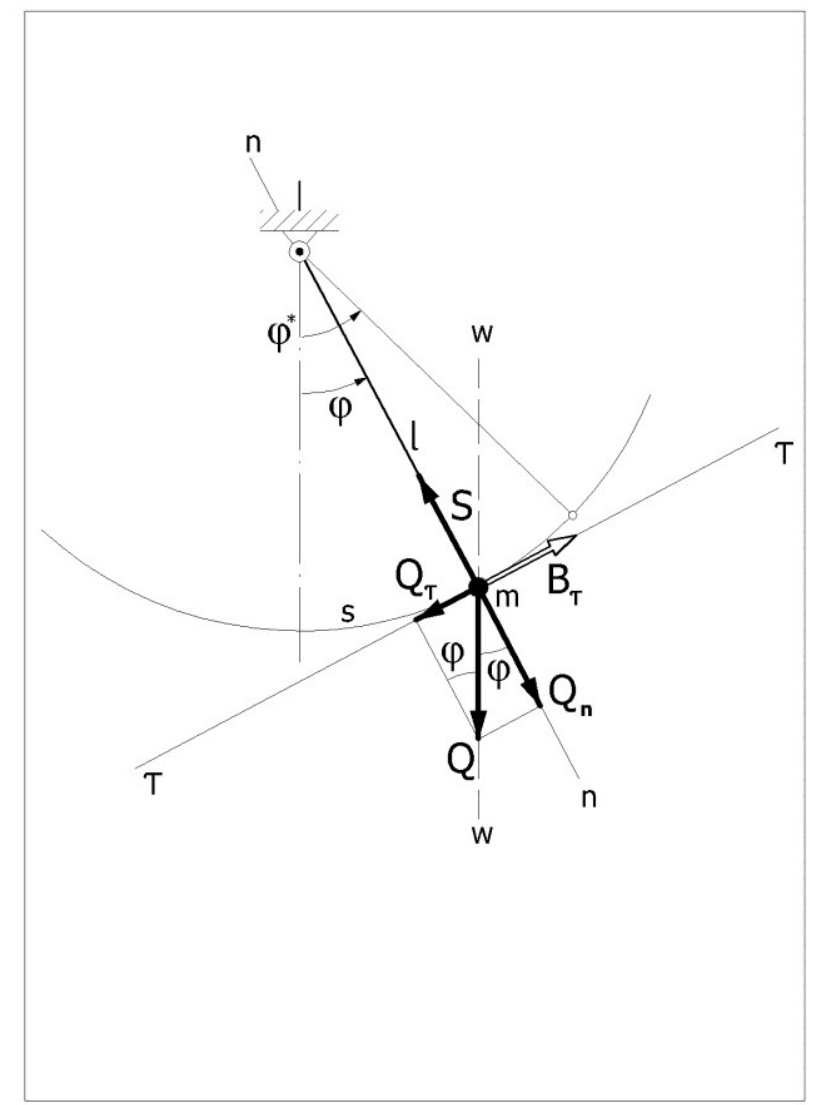

Fig. 1. Scheme of simple mathematical pendulum and the variable forces.

In that instantaneous intermediate position the weight undergoes different external actions. Forces of those reactions are different. One of those forces comes from the thread/string itself. It is the force of strain of thread, that is $S$. Second force results from the phenomenon of gravitation (gravity), so it is the force of gravity $Q$. That force has its location on a vertical direction $w-w$.

The existent theory of mathematical pendulum says that its oscillatory motion is caused by the force of gravity, i.e. $Q_{\tau}$, visible/noted on the direction tangent $\tau-\tau$ to the track of weight. As can be noticed, it is not balanced (not in equilibrium) by any other real force. In a 
normal direction to this track, i.e. $n-n$, the normal component $Q_{n}$ of the gravity force is balanced with the force of strain $S$ of the thread/string.

It is worth presenting now these concentrically running from the pendulum weight the particular forces (starry-like forces), vectors of the forces and present them in the form of polygons (Fig. 2). Conditions of the pendulum equilibrium (equilibrium of forces) are related to the closed polygon in the form of triangle (Fig. 2a) where, as can be seen, the force of gravity $Q$ is divided/decomposed into the components. The equivalent plan of particular forces (Fig. 2b) is related to this system where the closed polygon is the quadrilateral, and the force of gravity $Q$ is substituted by its two perpendicular, one to each other, components: normal $Q_{n}$, and tangent $Q_{\tau}$.

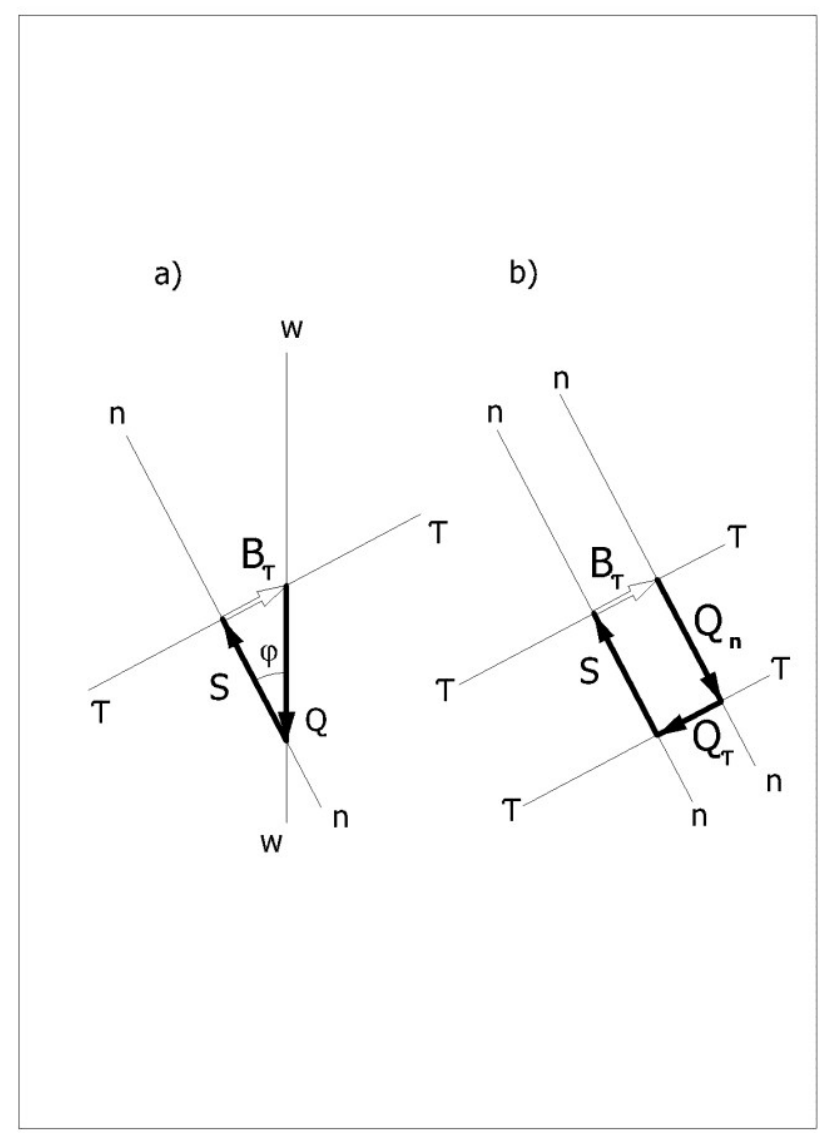

Fig. 2. Triangle (a) and tetragon of forces (b) in the case of mechanical reactions on weight of a simple mathematical pendulum.

The description of oscillatory motion of the considered pendulum results from the second Newton's law, and then, from the so called d'Alembert's rule [7]. It is commonly known that introduction of a fictional force (sic!) of gravity has been assumed in practice [8]. The aim of this strange action is an apparent balance of the force system in the direction of pendulum motion. Therefore, there will be two forces in such a fictional equilibrium: tangent component $Q_{\tau}$ of the gravity force and that fictional force of inertia $B_{\tau}$ (in Figure 2 that force is marked by a contour vector) [9].

Fictional force of inertia is expressed by the product of mass $m$ of a body and its tangent acceleration $a_{\tau}$ 


$$
B_{\tau}=m a_{\tau}
$$

It is known in turn that acceleration is the second derivative of the path length $s$ against time $t$, so that

$$
B_{\tau}=m \frac{d^{2} s}{d t^{2}}
$$

with the linear path length of weight in the direction of arc/bow of its track.

The mentioned tangent component $Q_{\tau}$ of the gravity force is expressed by the following formula:

$$
Q_{\tau}=m g \sin \varphi
$$

where symbol $g$ denotes the terrestrial acceleration (gravitation) [9].

A final equation of the force equilibrium (both real and fictional) has the following configuration

$$
m \frac{d^{2} s}{d t^{2}}=-m g \sin \varphi
$$

The sign (-), as theory explains, results from the fact that pendulum is deflected in the reverse direction to the force sense $Q_{\tau}$. The length $s$ of linear path of weight, resulting from the arc measure of angle, is determined by the dependence

$$
s=\varphi \cdot l
$$

where: $\varphi$-angle of pendulum deflection from the vertical direction (plumb-line), expressed as the arc measure (rad). Therefore, after regarding the dependence (5) in the formula (4),

$$
m \frac{d^{2} \varphi}{d t^{2}} \cdot l=-m g \sin \varphi
$$

Dividing both sides of equation (6) by $l$ leads to the record

$$
m \frac{d^{2} \varphi}{d t^{2}}=-m \frac{g}{l} \sin \varphi
$$

The quotient $g: l$ is further determined by symbol $\omega^{2}$

$$
\frac{g}{l}=\omega^{2}
$$

calling it as the circular or angular frequency. Therefore 


$$
m \frac{d^{2} \varphi}{d t^{2}}=-m \omega^{2} \sin \varphi
$$

The condition of small angles $(\sin \varphi \approx \operatorname{tg} \varphi \approx \varphi)$ allows to assume finally the formulation of pendulum motion, that is

$$
m \frac{d^{2} \varphi}{d t^{2}}=-m \omega^{2} \varphi
$$

or, after performing some determined algebraic transformations,

$$
\frac{d^{2} \varphi}{d t^{2}}+\omega^{2} \varphi=0
$$

This is, as can be noticed, a common differential equation of the second order. By solving it, one obtains a formula on the angular deflection of pendulum

$$
\varphi=\varphi^{*} \sin \omega t
$$

And now it is revealed that the symbol $\omega$ determines the real angular velocity, i.e.

$$
\omega=\frac{2 \pi}{T}
$$

where $T$ is the vibration period of mathematical pendulum (period of harmonic motion), that is time after which the motion is repeated. In other words, it is the double/twofold increased time of weight between the neighbouring turning points.

(That way one may get to the point of theoretical reasoning when the pendulum begins its rotating! Does that mean the pendulum is to travel the angular path corresponding with, or referred to, the multiplicity of round angle/perigon, that is the angle $2 \pi$ ? The commonly known definition of mathematical pendulum does not contain an information of such a kinetics. Hence, this kind of approach to the problem is senseless, absurd, preposterous, nonsensical, and completely illogic.)

Let us follow further consequences of such mystification reasoning. By comparing this magnitude, resulting from the formula (8)

$$
\omega=\sqrt{\frac{g}{l}}
$$

with the same magnitude, described by the formula (13), one obtains

$$
\frac{2 \pi}{T}=\sqrt{\frac{g}{l}}
$$

and further 


$$
T=2 \pi \sqrt{\frac{l}{g}}
$$

which is the formula on the period of vibrations of a simple mathematical pendulum. It is generally known that this formula serves to determine terrestrial acceleration/gravitation.

Reference [10] simplifies the description of motion of the simple mathematical pendulum. It is better clarified by the scheme serving to derive the equation of this motion (Fig. 3). Here, as can be seen, the path on the direction of circle (track of weight) has been switched to the horizontal direction. Thus the path arc has been substituted by an intercept/chord.

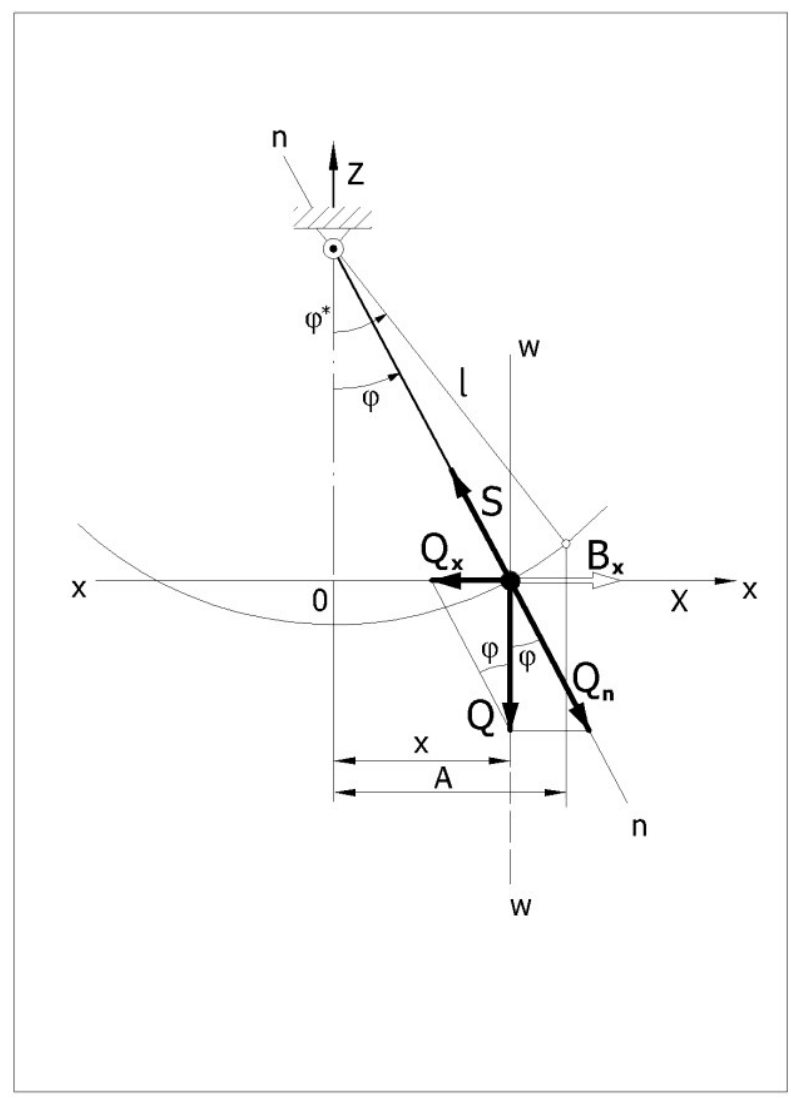

Fig. 3. Scheme used to derive the equation of pendulum motion with horizontal component of the force of gravity.

Therefore that means the force of gravity $Q$ (in vertical direction) should be decomposed onto horizontal component $Q_{x}$ (in the direction $x-x$ ) and the component $Q_{n}$, that is in the direction of pendulum deflection. Thus in the fictional equilibrium (resulting from the d'Alembert's rule [7]) there will be two forces: horizontal component $Q_{x}$ of the gravity force and a fictional force of inertia $B_{x}$ (this force has been denoted by a contour vector in Fig. 3).

It is also worth presenting that constellation of forces in the form of closed polygons (Fig. 4). For this, in the secondary simple mathematical pendulum, the direction of inertia leg in the triangle of forces (Fig. 4a) is horizontal. Quadrangle of forces (Fig. 4b) possesses one 
more such directed its component side; it relates with horizontal component of the gravity force.

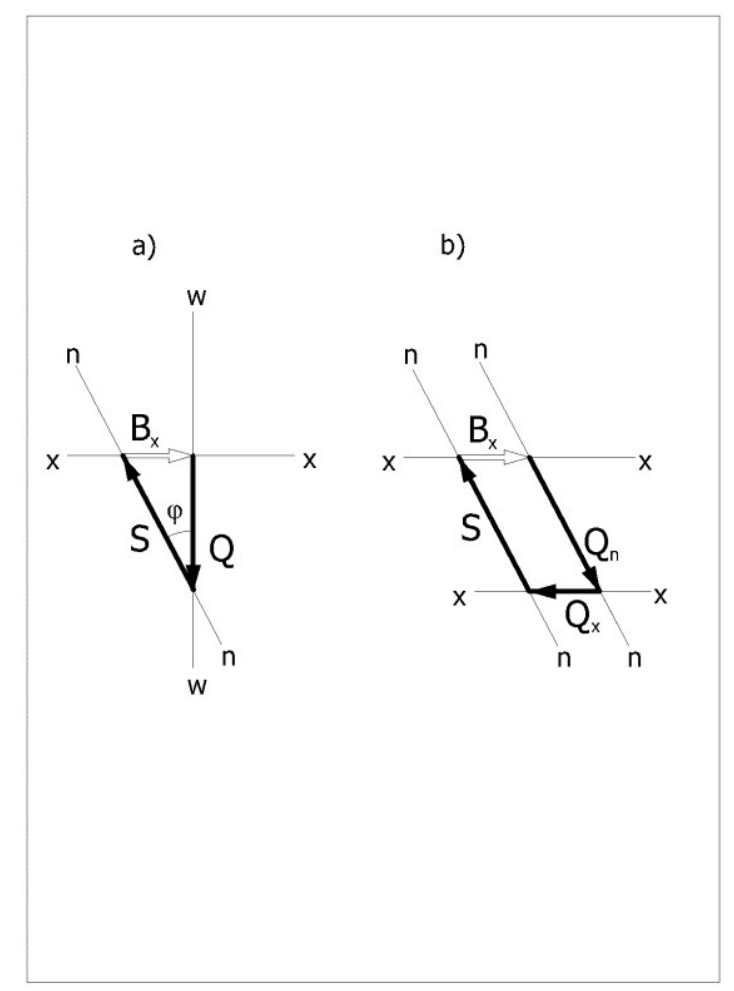

Fig. 4. Triangle (a) and tetragon of forces (b) in the case of mechanical reactions on weight of secondary simple mathematical pendulum.

That fictional force of inertia is expressed by the product of mass $m$ of a body and its horizontal acceleration $a_{x}$

$$
B_{x}=m a_{x}
$$

It is known in turn, that acceleration is the second derivative of the path $x$ against time $t$, so that

$$
B_{x}=m \frac{d^{2} x}{d t^{2}}
$$

The mentioned component $Q_{x}$ of the inertia force is expressed by the following formula:

$$
Q_{x}=m g \sin \varphi
$$

Finally, the equation of equilibrium of forces (both real and fictional) has the following configuration

$$
m \frac{d^{2} x}{d t^{2}}=-m g \sin \varphi
$$


Here again the theory explains that the sign (-) results from that the pendulum is deflected in the opposite direction to the sense of a real force, that is $Q_{x}$.

Regarding further that

$$
\sin \varphi=\frac{x}{l}
$$

the equation (20) may be recorded in the following form:

$$
m \frac{d^{2} x}{d t^{2}}=-m \frac{g}{l} x
$$

Assuming further (analogous as previously), that $g: l=\omega^{2}$, the formula (22) may be transformed to obtain

$$
\frac{d^{2} x}{d t^{2}}+\omega^{2} x=0
$$

The solution of this equation is the dependence

$$
x=A \sin \omega t
$$

where $A$ determines the amplitude (vastness) of the harmonic motion (maximum deflection of pendulum, or supremum of its deflections), and $x$ - instantaneous (which is intermediate) deflection of this pendulum.

Naturally, such an approach also leads to analogous formula on the period of pendulum vibrations. This is formula (16), resulting from definition (13) of the angular velocity, as well as the introduced assumption (14).

There is no need for mathematical development of solutions of the formulae (11) and (23) to a general and detailed solution of other, cosines form. Unreasonable is also that extended analysis of the subject, where the elliptical integrals are used. It is not required to prove the thesis that existent theory of the simple mathematical pendulum has a mystification nature. Instead, it is necessary to look at a physical nature of the investigated phenomenon.

\section{CONCLUSION}

Presented in the paper existent theory of a simple mathematical pendulum has many essential shortcomings. In the framework of critics of this theory there were particular insufficiencies discussed and described which confirm the formulated thesis on the theory having a mystification character. One may state all these drawbacks disqualify the existing theory.

There are many arguments proving against the existent theory of mathematical pendulum. Some illogical argumentations of these primary transactions have been indicated. It has surely been necessary to point out all shortcomings indicating the theory has been deeply rooted on the cognitive ground, functioning in the science for centuries. 


\section{References}

[1] P. Atkins, Galilei finger. Edit. House REBIS, Poznań 2006 (transl. from English), $1^{\text {st }}$ edition.

[2] S. Ziemba, Vibration analysis, PWN, Warszawa 1957, $1^{\text {st }}$ edition.

[3] Z. Osiński, Vibration theory, PWN, Warszawa 1978, $1^{\text {st }}$ edition.

[4] L. D. Landau, J. M. Lifszyc, Theoretical physics, Vol. 1: Mechanics, PWN, Warszawa 2006, $4^{\text {th }}$ edition (transl. from Russian).

[5] J. R. Taylor, Classical mechanics, Vol. 1. PWN, Warszawa 2006, $1^{\text {st }}$ edition (transl. from English)

[6] K. Chyla, Physics for students of secondary school. Edit. Office DEBIT, Bielsko Biała $1997,3^{\text {rd }}$ edition.

[7] Zdzisław Pluta, Tadeusz Hryniewicz, International Letters of Chemistry, Physics and Astronomy 7(2) (2013) 85-101.

[8] Zdzisław Pluta, Tadeusz Hryniewicz, International Letters of Chemistry, Physics and Astronomy 4 (2012) 8-16.

[9] Zdzisław Pluta, Tadeusz Hryniewicz, International Letters of Chemistry, Physics and Astronomy 5 (2012) 35-45.

[10] M. Jeżewski, Physics, PWN, Warszawa 1957, $5^{\text {th }}$ edition. 